\title{
Chemoenzymatic labeling of DNA methylation patterns for single- molecule epigenetic mapping
}

\author{
Tslil Gabrieli $^{1}$, Yael Michaeli ${ }^{1}$, Sigal Avraham ${ }^{1}$, Dmitry Torchinsky ${ }^{1}$, Matyas \\ Juhasz $^{2}$, Ceyda Coruh ${ }^{3}$, Nissim Arbib ${ }^{4}$, Zhaohui Sunny Zhou ${ }^{5}$, Julie A. Law ${ }^{3}$, \\ Elmar Weinhold*2, Yuval Ebenstein*1
}

\footnotetext{
${ }^{1}$ School of Chemistry, Center for Nanoscience and Nanotechnology, Center for Light-Matter Interaction, Raymond and Beverly Sackler Faculty of Exact Sciences, Tel Aviv University, Tel Aviv, Israel.

${ }^{2}$ Institute of Organic Chemistry, RWTH Aachen University, D-52056 Aachen Germany

${ }^{3}$ Plant Molecular and Cellular Biology Laboratory, Salk Institute for Biological Studies, La Jolla, CA, USA

${ }^{4}$ Department of Obstetrics and Gynecology, Meir Hospital, Kfar Saba, Israel \& Sackler Faculty of Medicine, Tel Aviv University, Tel Aviv, Israel.

${ }^{5}$ Department of Chemistry and Chemical Biology, and Barnett Institute of Chemical and Biological Analysis, Northeastern University, Boston, Massachusetts 02115, USA
}

Correspondence: Yuval Ebenstein, Tel: +972-3-640-8901. Fax: +972-3-6405794. E-mail: uv@post.tau.ac.il; Elmar Weinhold, Tel: +49-241-8094044. Fax: +49-241-8092528. E-mail: elmar.weinhold@oc.rwth-aachen.de

\begin{abstract}
DNA methylation, specifically, methylation of cytosine (C) nucleotides at the 5-carbon position $(5-\mathrm{mC})$, is the most studied and among the most significant epigenetic modifications. Here we developed a chemoenzymatic procedure to fluorescently label non-methylated cytosines in the $\mathrm{CpG}$ context allowing epigenetic profiling of single DNA molecules spanning hundreds of thousands of base pairs. For this method, a CpG methyltransferase was used to transfer an azide to cytosines from a synthetic $S$ adenosyl-1-methionine cofactor analog. A fluorophore was then clicked onto the DNA, reporting on the amount and position of non-methylated CpGs. We found that labeling efficiency was increased two-fold by the addition of a nucleosidase that degrades the
\end{abstract}


inactive by-product of the azide-cofactor after labeling, and prevents its inhibitory effect. We first used the method to determine the decline in global DNA methylation in chronic lymphocytic leukemia patients and then performed whole genome methylation mapping of the model plant Arabidopsis thaliana. Our genome maps show high concordance with published methylation maps produced by bisulfite sequencing. Although mapping resolution is limited by optical detection to 500-1000 base pairs, the labeled DNA molecules produced by this approach are hundreds of thousands of base pairs long, allowing access to long repetitive and structurally variable genomic regions.

\section{INTRODUCTION}

DNA methylation is an epigenetic mark that plays a major regulatory role in transcription, gene regulation, and disease. 5-methylcytocine (5-mC) is conserved among plants and mammals, and its precise genomic patterns are crucial for development. In mammals, 5-mC occurs most often in the context of $\mathrm{CpG}$ dinucleotides and, in the human genome, most CpGs are highly methylated (between 70-80\%). However, in specific locations, mainly high density $\mathrm{CpG}$ islands (CGI), they remain mostly non-methylated. The majority of CGIs $(\sim 70 \%)$ are located at gene promoters and the methylation status of these regions are known to regulate gene expression ${ }^{1,2,3}$. In cancer, large-scale changes in methylation levels are observed and can include both genome-wide hypomethylation as well as more localized hypermethylation, mainly of tumor suppressor genes that are often silenced in cancer ${ }^{4,5,6,7,8}$.

Methylation is established by a diverse family of methyltransferases (MTases). These enzymes utilize $S$-adenosyl-L-methionine (AdoMet) as the methyl donor, forming methylated DNA and $S$-adenosyl-L-homocysteine (AdoHcy) as a by-product ${ }^{9}$. Various approaches have been developed to specifically profile cytosine DNA methylation, both on a global level and at base pair resolution. The gold standard technique is bisulfite sequencing (called BS-seq (Cokus et al. ${ }^{10}$ ) or methylC-seq (Lister et al. ${ }^{11}$ )) depending on the methods used), which relies on chemical conversion of unmodified cytosines to uracil, while leaving methylated cytosines unconverted ${ }^{10,12}$. After PCR amplification and sequencing, the originally methylated cytosines are read as $\mathrm{C}$ while the unmodified cytosines are read as T. However, this method can suffer from several limitations, such as high DNA degradation and potential biases due to the amplification process. Short read sequencing also adds several drawbacks and mainly the limitation in characterizing large variable and repetitive regions, as well as population averaging 
of the data which masks cell-to-cell variation ${ }^{13}$. Single-cell bisulfite sequencing may profile individual methylomes ${ }^{14-15}$ but is still limited by short read sequencing and thus is not able to fully characterize highly repetitive regions. Additionally, the technique suffers from low sampling of the individual genomes, thus requiring sequencing the genomes of thousands of individual cells ${ }^{16}$.

Third generation sequencing approaches, including single-molecule, real-time (SMRT) sequencing (Pacific Biosciences Inc.) and nanopore sequencing (Oxford Nanopore Technologies Ltd.), are able to detect methylated cytosines directly ${ }^{17-21}$. However, the accuracy of epigenetic analysis on these platforms is still lacking and whole genome analysis is challenging ${ }^{22}$.

Whole-genome epigenetic profiling by optical genome mapping (Bionano Genomics Inc.) is a new addition to the epigenetic mapping toolbox. This technology is based on stretching long genomic DNA fragments for optical imaging. The DNA is labeled with two colors, one is used for aligning the molecules to the reference genome and the other reports on the epigenetic content of the molecules ${ }^{23-25}$. This technique outputs extremely long, single-molecule data (N50 200 kbp) that allows profiling of large variable and repetitive regions ${ }^{26}$.

Our group developed Reduced representation Optical Methylation mapping (ROM), as a method for the labeling and detection of non-methylated $\mathrm{CpG}$ sites using the bacterial MTase, M.TaqI, and a cofactor analogue ${ }^{24}$. Recently, this long-read methylation data was analyzed at a single-cell level for the first time. The methylation status of promoters and their distal enhancers, simultaneously imaged on the same long DNA molecules, served to accurately deconvolve cell-type mixtures and subpopulations within a sample ${ }^{27}$. The non-methylation labeling scheme relies on the transfer of a fluorescent molecule from a synthetically modified form of the native methylation cofactor AdoMet. The enzyme is "tricked" into transferring the extended chemical group with a fluorophore instead of the natural methyl group, resulting in fluorescence at nonmethylated CpG sites. However, M.TaqI (recognition site: TCGA) only samples about $6 \%$ of human $\mathrm{CpG}$ sites and thus can fail to capture vital epigenetic information.

Here we present a chemoenzymatic labeling approach for the detection of nonmethylated $\mathrm{CpGs}$ as a means for a global quantification of methylation and for genomic methylation mapping. This is achieved by utilizing the CpG-specific MTase M.SssI from the bacteria Spiroplasma sp. strain MQ-1 that naturally transfers a methyl group to the fifth position of cytosines in $\mathrm{CpG}$ dinucleotides ${ }^{28,29}$. While M.SssI has the 
potential to label all non-methylated CpGs, it is not able to utilize modified AdoMet cofactors. However, an engineered variant of M.SssI (Q142A/N370A) is able to transfer an azide group to non-methylated $\mathrm{CpG}$ sites by processing an azide-modified cofactor AdoYnAzide (Ado-6-azide). This engineered M.SssI (eM.SssI) was used by Kriukiene et al. for non-methylation sequencing ${ }^{30}$. However, the affinity capture on streptavidin-modified beads showed a $20-30 \%$ capture efficiency, indicating poor labeling yield which is insufficient for single-molecule optical mapping. To overcome the low labeling efficiency, we employed the enzyme 5-methylthioadenosine/Sadenosylhomocysteine nucleosidase (MTAN, EC 3.2.2.9) that catalyzes hydrolysis of the glycosidic bond in AdoHcy. Thus, MTAN degrades the inactive cofactor byproduct, AdoHcy, formed from the natural cofactor AdoMet or synthetic cofactor analogues, like AdoYnAzide (Figure 1A). In general, AdoHcy is a well-known product inhibitor for MTases ${ }^{31}$. Lowering its concentration by addition of MTAN effectively drives the reaction towards increased labeling efficiency (Figure 1A). The azidemodified DNA can then be covalently labeled by a dibenzocyclooctyne-cy5 (DBCOcy5) fluorophore in a strain-promoted azide-alkyne cycloaddition (SPAAC, copper-free click chemistry reaction), to specifically label non-methylated $\mathrm{CpG}$ sites. Thus, the increased efficiency of the eM.SssI / MTAN combination enabled generation of optical maps that present genome-wide epigenetic status of $\mathrm{CpG}$ sites with single-molecule sensitivity.

Here, we apply this labeling strategy to human peripheral blood mononuclear cells (PBMCs), emphasizing the ability to quantify the methylation status of $\mathrm{CpG}$ sites in healthy vs. cancer patients with high sensitivity. We further demonstrate whole-genome methylation mapping of the model plant $A$. thaliana. Our approach allows simultaneous genetic and epigenetic characterization of long streches of DNA at the single molecule level, and potentially permits studying variations between single cells.

\section{RESULTS AND DISCUSSION}

\section{Efficient labeling of non-methylated CpGs}

Single-molecule epigenomic mapping requires highly efficient labeling in order to extract the maximum information from the studied sample. We hypothesized that during labeling by eM.SssI the cofactor by-product AdoHcy could reduce the overall 
labeling efficiency by occupying the cofactor binding pocket and preventing additional labeling cycles (Figure 1A). Thus, we sought to enzymatically degrade this by-product by the addition of recombinant MTAN ${ }^{32}$. To test the effect of MTAN on the labeling efficiency of eM.SssI, we labeled non-methylated $\lambda$ DNA using eM.SssI, AdoYnAzide, and DBCO-cy5, with or without MTAN (Figure 1B \& Figure S1). Figure 1C shows the relative fluorescence intensity of $\lambda$ DNA labeled with or without MTAN. Average labeling intensity increased two-fold with the addition of MTAN to the reaction (Figure 1C, Figure S2) and reached a level that enables reliable single-molecule analysis and epigenetic genome mapping.

A
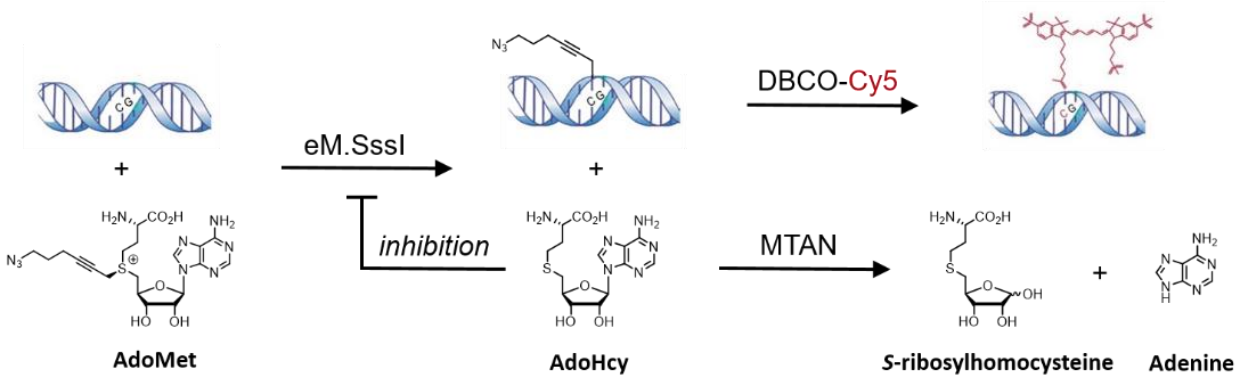

B
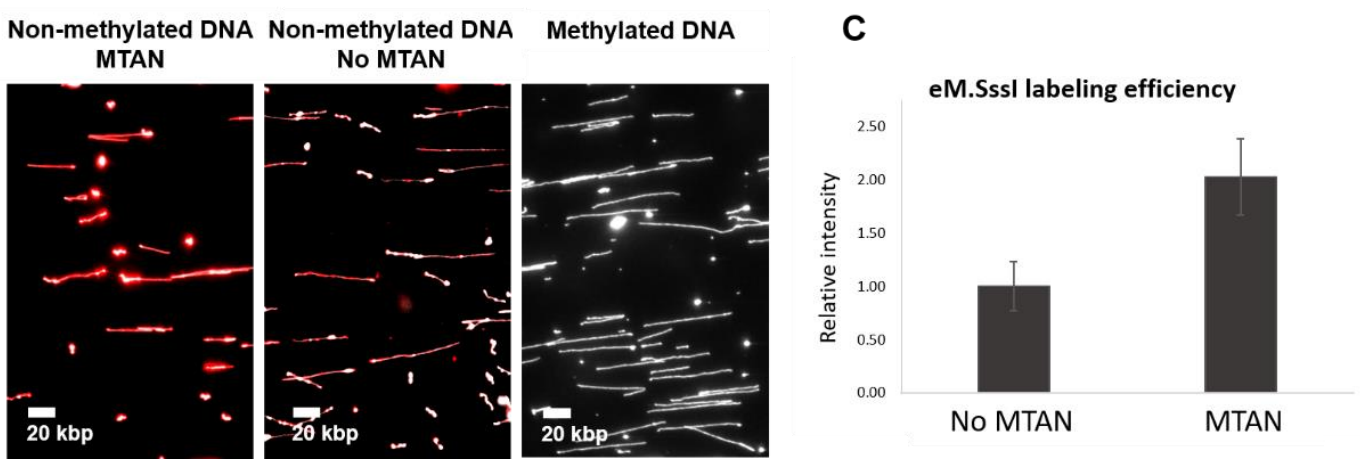

Figure 1. A. eM.SssI catalyzes the addition of AdoYnAzide to non-methylated CpGs. DBCOcy5 is then attached by click chemistry to the azide-modified CpGs. Following the transfer of the azide-containing side chain by eM.SssI, the remaining cofactor by-product AdoHcy serves as a substrate for MTAN that hydrolyzes it to adenine and $S$-ribosylhomocysteine. In the absence of MTAN, AdoHcy accumulates and can bind to eM.SssI, inhibiting its activity. B. A representative field of view of non-methylated $\lambda$ DNA labeled with eM.SssI, in the presence (Left) or absence (Middle) of MTAN. Methylated DNA was labeled as control (Right). GrayDNA backbone, red- non-methylated CpG labels. C. Global quantification (calculated by dividing the total intensity of the $\mathrm{CpG}$ label by the total length of the DNA) of non-methylated CpGs labelled with eM.SssI, with or without MTAN. 


\section{Global methylation quantification}

One of the hallmarks of cancer is global reduction in genomic methylation levels ${ }^{33}$. As a first step in evaluating our optical mapping technique for epigenetic profiling, we quantified the genome-wide methylation level of PBMCs from a patient diagnosed with chronic lymphocytic leukemia (CLL) and a healthy donor. Both healthy and patient DNA were stretched in nanochannel array chips, imaged, and the fluorescence from the non-methylated $\mathrm{CpG}$ labels was detected (Figure 2A). Fluorescence intensity along the labeled DNA molecules was automatically measured to determine the relative difference in labeling ${ }^{34}$. The relative global methylation level was measured by summing the overall red intensity (i.e. non-methylated cytosines in the $\mathrm{CpG}$ context) along the DNA molecules, normalized to the total DNA length measured for each sample (Figure 2B). When comparing genomic DNA from a patient and a healthy donor, the cancer cells show a global reduction in methylation levels indicated by a higher fluorescence signal along the DNA (Figure 2B). Specifically, the signal intensity from non-methylated CpGs increased by 2.3 -fold in the CLL sample compared to the healthy control, indicating global hypomethylation in the CLL sample as previously reported (supplementary files $2 \& 3)^{35}$. Incorporation of our novel chemoenzymatic labeling method into single molecule optical mapping allows us to distinguish global DNA methylation differences between samples, opening up potential application of this technique for DNA methylation research and medical diagnostics.
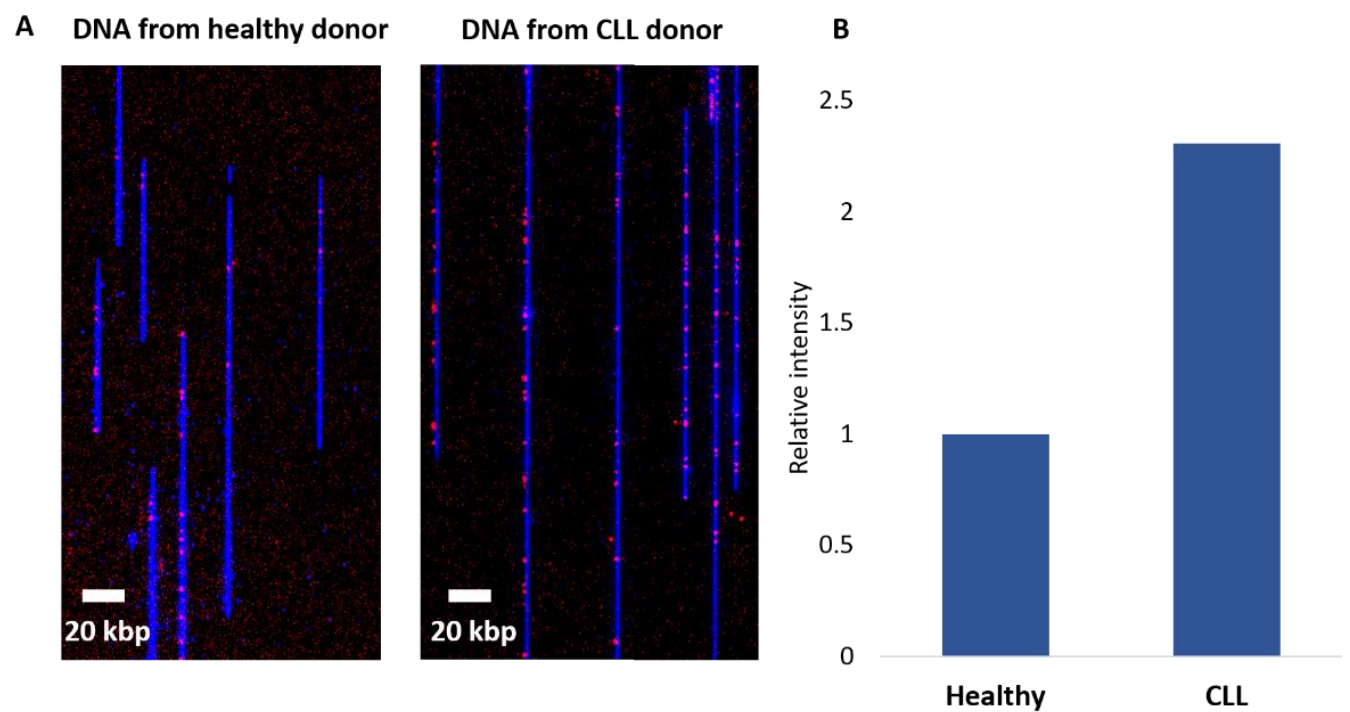
Figure 2. Methylation quantification in human peripheral blood mononuclear cells (PBMCs) from a healthy and CLL patient donor. A. Representative images of stretched DNA in nanochannel array chips from both samples: DNA backbone in blue and epigenetic labels in red (more red signal denotes less DNA methylation at $\mathrm{CpG}$ sites). B. Global quantification of the methylation signal intensity along the DNA molecules. Intensity of the healthy sample was normalized to 1 .

\section{Genomic mapping of DNA methylation}

We next attempted to map the $\mathrm{CpG}$ methylation profile in the well-characterized genome of the model plant, Arabidopsis thaliana. This genome is composed of five chromosomes with a total size of $\sim 135 \mathrm{Mbp}$. The relatively small genome allowed us to test mapping feasibility while avoiding the cost and computational complexity of analyzing the human genome. In A. thaliana DNA methylation commonly occurs in all sequence contexts (CG, $\mathrm{CHG}$ and $\mathrm{CHH}$, where $\mathrm{H}=\mathrm{A}$, $\mathrm{T}$ or $\mathrm{C})^{10,12}$ and the presence of methylation at transposons and repeats, as well as some protein coding genes, is associated with gene silencing and genome stability ${ }^{12,36,37}$. A. thaliana DNA was extracted, non-methylated CpGs were labeled using our modified M.SssI system, and genomic mapping was performed by using nickase labeling to create a barcode of specific sequence motifs. The dual-labeled DNA molecules were then electrokinetically forced into nanochannels and imaged on the Bionano Genomics (Inc) Irys system, allowing the detection of fluorescent labeling patterns (Figure 3A and 3B). We detected 34,866 DNA molecules longer than $100 \mathrm{kbp}$ and 28,917 were successfully aligned to the TAIR10 A. thaliana reference genome (Figure S3). The analysis resulted in an average mapping depth of $47 x$, covering $~ 97 \%$ of the genome with a minimum coverage of 15 molecules. The intensity profile of the epigenetic labels for each molecule was created by Irys Extract ${ }^{38}$, followed by calculation of normalized average intensity across all molecules aligned to the same region (Figure 3C). 


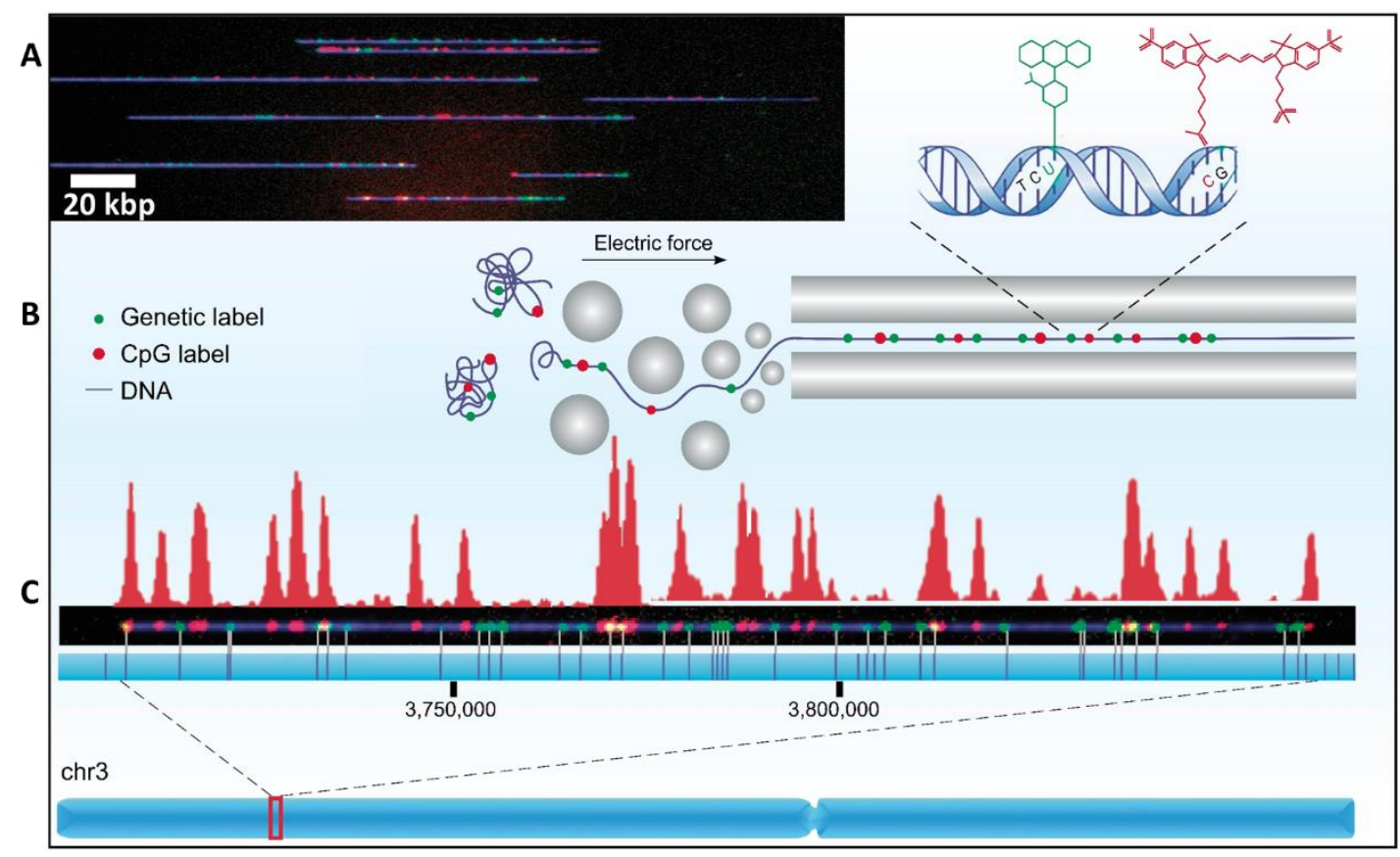

Figure 3. Optical methylation mapping scheme. A. A representative image of stretched DNA molecules in a nanochannel array. DNA backbone in blue (YOYO-1), genetic labels in green (Atto-532) and non-methylated $\mathrm{CpG}$ labels in red (cy5). B. Schematic representation of the fluorescently labeled molecules unraveled and extended in a nanochannel array by using an electric field. C. A $150 \mathrm{kbp}$ DNA molecule aligned to chromosome 3 by the green genetic labels. The vertical lines on the blue strip represent the theoretical positions of genetic labels in the reference genome. The fluorescence intensity pattern representing the levels of nonmethylated $\mathrm{CpG}$ sites along the molecule is presented in red above the molecule.

\section{Optical methylation mapping is well correlated with bisulfite sequencing}

To further evaluate methylation mapping, a genome-wide correlation of publiclyavailable bisulfite sequencing data ${ }^{36}$ with epigenetic optical mapping was calculated. Since our method captures signal from non-methylated CpGs, we present the genomewide bisulfite sequencing data as the non-methylated fraction of CpGs. To overcome scaling and resolution differences between the two methods, each non methylated $\mathrm{CpG}$ from the bisulfite sequencing data was padded $+/-500 \mathrm{bp}$ and summed across the genome in $10 \mathrm{kbp}$ bins to generate a "methylation score" that corresponds to the amount of non-methylated $\mathrm{CpG}$ sites in each bin (i.e. a higher score equates to more nonmethylated $\mathrm{CpG}$ sites,). This binned data was then compared to the average intensity profile of optical epigenome mapping. First, optical data was normalized for coverage variations by dividing the summed local intensity by the local coverage to obtain a 
fluorescence intensity score in $1 \mathrm{kbp}$ bins across the genome. The normalized intensity score in each bin was then divided by the global maximum intensity (e.g. the $1 \mathrm{kbp}$ bin with the highest fluorescence intensity score), setting the relative intensity data between 0-1. As presented in Figure 4A, the optical mapping data nicely correlate with the genome-wide bisulfite sequencing data with a Spearman correlation coefficient of 0.745 (supplementary file 4). In addition, a global view of the A. thaliana genome showing the levels of non-methylated $\mathrm{CpG}$ sites illustrates the correlation between the optical mapping and genome-wide bisulfite sequencing data (Figure 4B). The centromeres of the A. thaliana genome are enriched in methylated CpGs and are thus seen as dips in the optical graphs at the centromeric positions (low signal denotes high methylation, Figure 4B). The lower panel of Figure 4B shows a zoomed-in region in Chr1 illustrating the positive correlation between the two methods. The finer details in the blue track highlights the higher resolution of the genome-wide bisulfite sequencing data. Nevertheless, the long molecules observed by optical mapping (>100 kbp) allowed us to assemble a reliable and highly contiguous epigenetic profile of the $A$. thaliana genome.
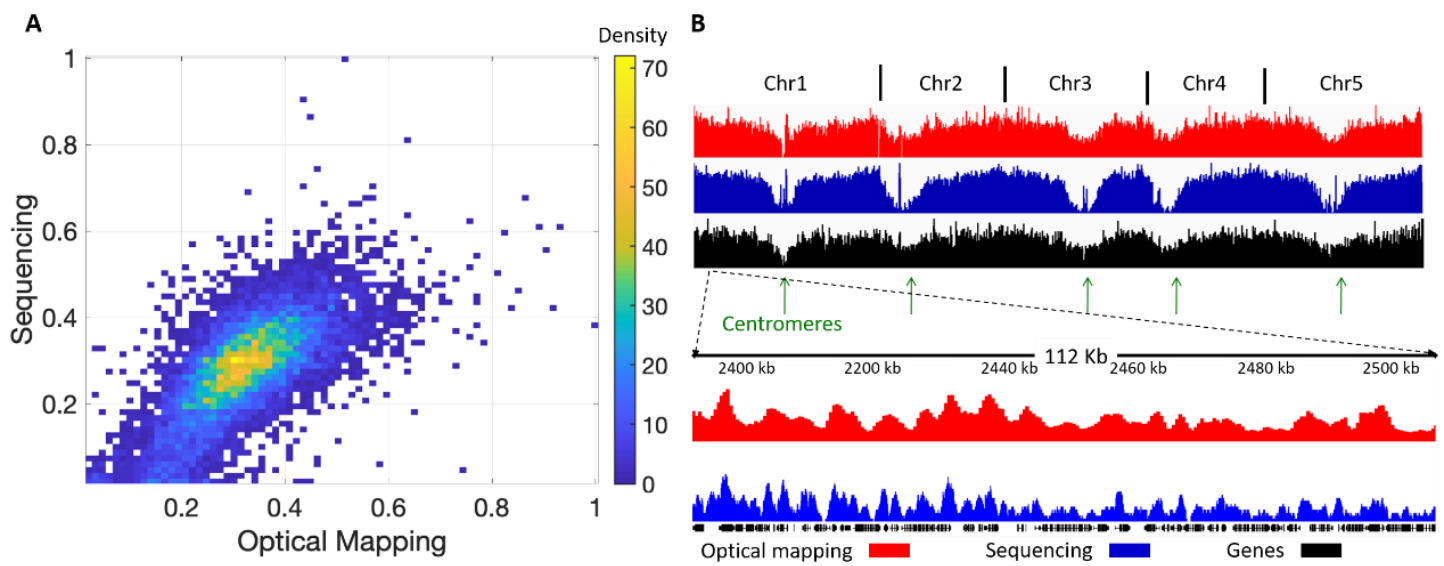

Figure 4. Global correlation between genome-wide bisulfite sequencing and methylation optical mapping. A. Density scatter plot representing the global correlation between genomewide bisulfite sequencing and optical mapping in $10 \mathrm{kbp}$ bins. B. Global view of the optical methylation profiles (relative intensity) in red and the genome-wide bisulfite sequencing data (methylation score) in blue, both showing the levels of non-methylated $\mathrm{CpG}$ sites. For reference, the genes are shown in black and the centromeres are indicated by green arrows. The bottom panel presents a zoomed-in view of a selected region. 


\section{Optical patterns correlate with genome-wide bisulfite sequencing at siRNA clusters and gene bodies}

In A. thaliana, DNA methylation at transposons and repeats is guided by short interfering RNAs (siRNAs) that recruit the DNA cytosine MTase, DOMAINS REARRANGED METHYLTRANSFERASE 2 (DRM2), via protein/RNA complexes $^{39,40}$. Since the $24 \mathrm{nt}$ siRNAs guide where DNA methylation occurs in the plant genome, including methylation in the $\mathrm{CpG}$ context, we checked if the $\mathrm{CpG}$ methylation patterns from bisulfite sequencing data correlates as expected with the optical data at sites that produce $24 \mathrm{nt}$ siRNAs (i.e. sites producing $24 \mathrm{nt}$ siRNAs should have lower levels of non-methylated $\mathrm{CpG}$ sites compared to surrounding regions). Methylation scores from genome-wide bisulfite sequencing data (supplementary file 5) and relative intensity values from optical mapping data were calculated $10 \mathrm{kbp}$ upstream and downstream relative to the $24 \mathrm{nt}$ siRNA clusters (position 0$)^{41}$. These data were combined into a single plot representing the methylation profile around these regions (Figure 5A). The methylation score from bisulfite sequencing data (blue) shows a sharp dip around position 0, indicating highly methylated DNA around 24nt siRNA clusters. The relative intensity values from the optical mapping data (red) correlates well with this trend, although at lower resolution, as expected from the optical diffraction limit.

As negative controls, we examined optical mapping data over primary miRNA loci and several trans-acting siRNA loci (TAS loci) which are not related to DNA methylation (Figure S4, supplementary file 5). As expected, the intensity profile across the miRNA precursor and TAS loci did not have a specific pattern around the cluster center.

In addition to DNA methylation in all sequence contexts at transposons and repeats, some gene bodies are methylated specifically in $\mathrm{CpG}$ context in the A. thaliana genome and this methylation is distributed away from the 5 region of genes ${ }^{10}$. Thus, we also compared the methylation profiles across gene bodies from genome-wide bisulfite sequencing and optical mapping (supplementary file 6). Figure 5B illustrates the profiles across the gene bodies and $1.5 \mathrm{kbp}$ upstream of the transcription start site (TSS) and downstream from the transcription end site (TES). Again, the two datasets show the expected modulation in methylation along the gene, with sequencing data displaying higher resolution (as seen downstream of the TES). While lacking in resolution, optical mapping has the advantage of visualizing intact genes as well as 
extremely long DNA molecules that are able to capture both large-scale methylation patterns, and methylation across highly variable regions that are difficult to map by short-read sequencing.
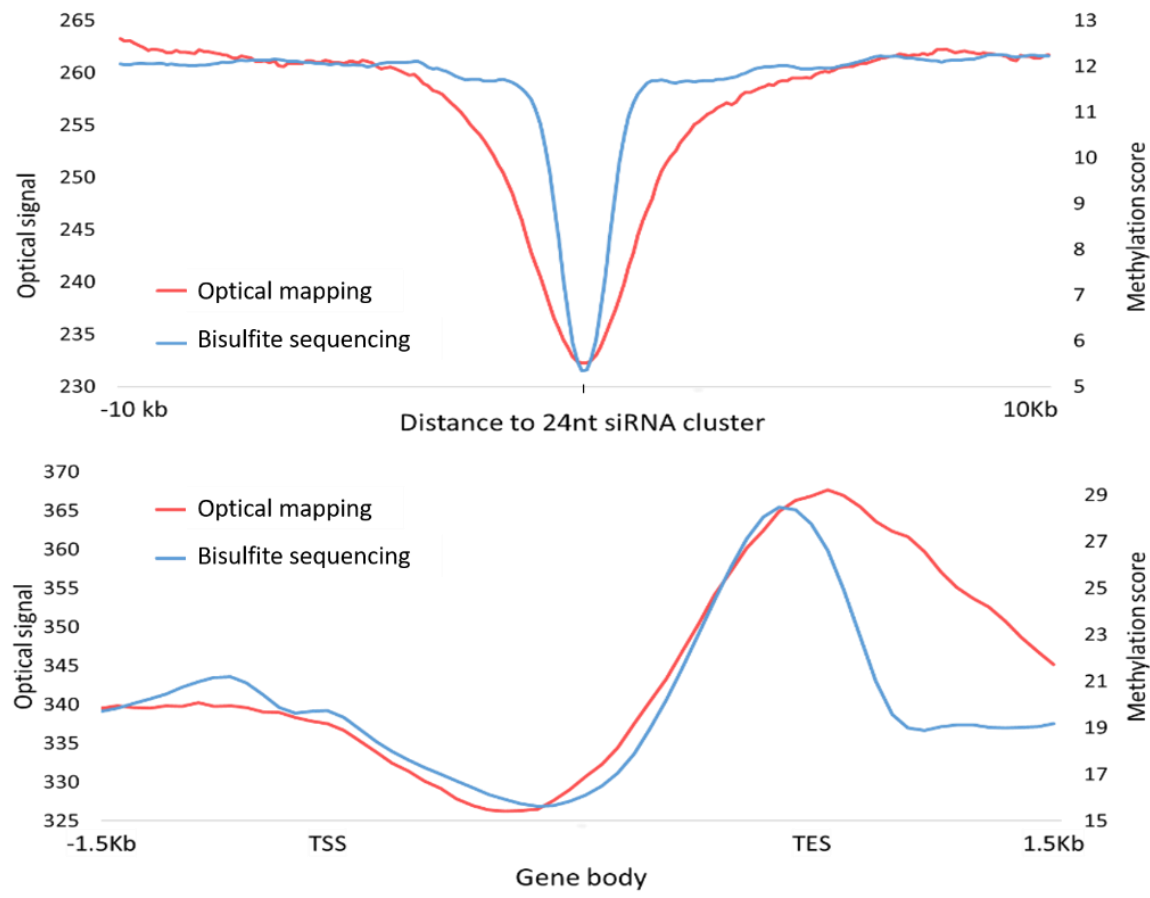

Figure 5. Methylation profiles at 24nt siRNA producing loci and across gene bodies. Genomewide bisulfite sequencing data in blue \& optical mapping data in red. A. 24nt siRNA loci. B. Methylation profile across gene bodies and $1.5 \mathrm{kbp}$ upstream and downstream to the transcription start site (TSS) and the transcription end site (TES), respectively.

\section{CONCLUSIONS}

In this study we use the DNA methyltransferase eM.SssI and a synthetic cofactor together with the nucleosidase MTAN to fluorescently label non-methylated $\mathrm{CpG}$ dinucleotides. The addition of MTAN to the reaction dramatically increased the labeling efficiency of eM.SssI, making the method compatible with single-molecule analysis. Application of this labeling approach to optical genome mapping enables genome-wide methylome profiling, offering long-range information that can span long variable regions and repeats ${ }^{23,24}$. We note that this concept may be similarly utilized to map other genomic features such as other epigenetic modifications and DNA damage lesions ${ }^{23,24,42-45}$. 
Global methylation changes can be quantified by integrating the optical methylation signal for a given amount of DNA (Figure 2). Assessment of whole genome methylation levels may serve as a diagnostic tool that can distinguish between patients with healthy or malignant cells. It is long known that patients with B-cell chronic lymphocytic leukemia (CLL) experience hypermethylation in specific genes ${ }^{46}$ while their global methylation levels decrease ${ }^{35}$. This hypomethylation can be detected by the presented optical labeling scheme with very high sensitivity, emphasized by the distinct differences in labeling between the healthy and CLL patient (Figure 2B).

The generation of whole genome methylation maps by means of optical mapping was demonstrated here on the model plant A. thaliana and found to correlate well with genome-wide bisulfite sequencing data. As many plant genomes are highly repetitive, optical mapping is becoming a routine method for genomic characterization in plants ${ }^{47-}$ ${ }^{49}$. By utilizing this new approach, it is now possible to access methylation information in repetitive or otherwise challenging regions. While optical mapping resolution is limited by the optical diffraction limit to around $1 \mathrm{kbp}$, we increased the effective resolving power of the technique by using fluorescence intensity in addition to genomic location. Thus, $\mathrm{CpG}$-rich regions, where more than one $\mathrm{CpG}$ site resides within the optical spot, can now be characterized by signal intensity that reports on labeling density, i.e., the actual number of labeled $\mathrm{CpG}$ sites at a given fluorescent spot. Future extension of the method to the human genome may facilitate early diagnosis of malignant transformations related to DNA methylation.

\section{METHODS}

\section{Human Subjects}

The healthy donor sample used in this study was collected with informed consent for research use and approved by the Tel-Aviv University and Meir medical center ethical Review Boards, in accordance with the declaration of Helsinki. The PBMCs from a CLL donor were purchased from BioServe. 


\section{High-molecular-weight DNA extraction}

High molecular weight (HMW) DNA was extracted for optical mapping from $1 \mathrm{~g}$ of 68 weeks old whole plant tissue, before flowering using the BioNano Genomics Fix'n'Chop protocol with some modifications. Briefly, no formaldehyde was used, chopping was done only with lab blender and no razor blade was used, $7.5 \%$ of 2Mercaptoethanol was used. Following the final wash, nuclei pellet was resuspended in cell suspension buffer (CHEF mammalian DNA extraction kit, Bio-Rad) and incubated at $43{ }^{\circ} \mathrm{C}$ for $10 \mathrm{~min} .2 \%$ low melting agarose (CleanCut agarose, Bio-Rad) was melted at $70{ }^{\circ} \mathrm{C}$ followed by incubation at $43^{\circ} \mathrm{C}$ for $10 \mathrm{~min}$. Melted agarose was added to the resuspended cells at a final concentration of $0.75 \%$ and mixed gently. The mixture was immediately cast to a plug mold and plugs were incubated at $4{ }^{\circ} \mathrm{C}$ until solidified. For human peripheral blood mononuclear cells (PBMCs), peripheral blood of a healthy donor was isolated by density gradient centrifugation using Ficoll Paque Plus (GE Healthcare) according to manufacturer's instructions. Plugs were prepared according to Plug Lysis protocol (BioNano Genomics). Briefly, $1 \times 10^{6}$ cells were washed twice with PBS and mixed with 2\% low melting agarose as described above. All plugs were incubated twice ( 2 hours incubation followed by an overnight incubation) at $50{ }^{\circ} \mathrm{C}$ with freshly prepared $167 \mu \mathrm{l}$ Proteinase K (Qiagen) in $2.5 \mathrm{ml}$ lysis buffer (BioNano Genomics) with occasional shaking. Next, plugs were incubated with $50 \mu 1$ RNase (Qiagen) in $2.5 \mathrm{ml} \mathrm{TE}\left(\mathrm{pH}\right.$ 8) for 1 hour at $37{ }^{\circ} \mathrm{C}$ with occasional shaking. Plugs were washed three times by adding $10 \mathrm{ml}$ wash buffer (10 mM Tris, $\mathrm{pH} \mathrm{8,50} \mathrm{mM} \mathrm{EDTA),}$ manually shaking for 10 seconds and discarding the wash buffer before adding the next wash. Plugs were then washed four times by adding $10 \mathrm{ml}$ wash buffer and shaking for $15 \mathrm{~min}$ on a horizontal platform mixer at $180 \mathrm{rpm}$ at room temperature. Following washes, plugs were stored at $4{ }^{\circ} \mathrm{C}$ in wash buffer or used for labeling. In order to extract high molecular weight DNA, plugs washed three times in TE $\mathrm{pH} 8$ with shaking on a horizontal platform as explained, were melted for $2 \mathrm{~min}$ at $70^{\circ} \mathrm{C}$, followed by $5 \mathrm{~min}$ incubation at $43{ }^{\circ} \mathrm{C}$. Next, 0.4 units of Gelase (Thermo Fisher) were added and the mixture was incubated for $45 \mathrm{~min}$. Viscous DNA was gently pipetted and incubated at room temperature overnight in order to achieve homogeneity. DNA concentration was determined using Qubit BR dsDNS assay. 


\section{Genetic barcoding and non-methylated CpG labeling}

Genetic barcodes were produced by nick translation, a modified version of Irys prep NLRS protocol (Bionano Genomics). Briefly, 300 ng DNA were nicked with 10 units of Nt.BspQI (NEB) at specific sequence motifs $\left(\mathrm{GCTCTTCN}^{\wedge}\right)$ in the presence of $1 \mu \mathrm{l}$ $10 \mathrm{X}$ buffer $3.1(\mathrm{NEB})$ for $2 \mathrm{~h}$ at $50^{\circ} \mathrm{C}$ and ultra-pure water to a volume of $10 \mu \mathrm{l}$. For DNA labeling, 15 units of Taq DNA polymerase were incubated with $200 \mathrm{nM}$ of dATP $\mathrm{dGTP} \mathrm{dCTP}$ (Sigma) and atto-532-dUTP (Jena Bioscience) in the presence of $1.5 \mu 1$ of 10X thermopol buffer and ultra-pure water to a volume of $15 \mu l$, the reaction was incubated at $72{ }^{\circ} \mathrm{C}$ for $1 \mathrm{~h}$. Nicks were then ligated for $30 \mathrm{~min}$ at $37^{\circ} \mathrm{C}$ using 4 units of Taq DNA ligase (NEB) in the presence of $0.5 \mu$ l thermopol buffer, $20 \mu \mathrm{M}$ dNTPs

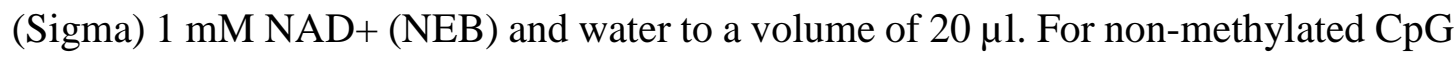
labeling (mTAG labeling) $300 \mathrm{ng}$ of nick-labeled DNA were incubated overnight at 37 ${ }^{\circ} \mathrm{C}$ with $4.3 \mu \mathrm{g}$ of the $\mathrm{CpG}$-specific cytosine-C5 MTase M.SssI double mutant Q142A/N370A (eM.SssI) ${ }^{26}$ in the presence of $80 \mu \mathrm{M}$ cofactor analogue AdoYnAzide ${ }^{50}$ and $3 \mu 1$ 10X M.SssI reaction buffer (Thermo fisher/10 mM Tris-HCL, $50 \mathrm{mM} \mathrm{NaCl}$, $1 \mathrm{mM}$ DTT, pH 7.9), in a total reaction volume of $30 \mu 1$. The reaction was supplemented with $2 \mu \mathrm{M}$ 5'-methylthioadenosine/S-adenosylhomocysteine nucleosidase (MTAN) and after $2 \mathrm{~h}$ of incubation, a spike-in of $1.1 \mu \mathrm{g}$ of the eM.SssI and $2 \mu \mathrm{M} \mathrm{MTAN}^{51,52}$ was added to the reaction to further improve labeling efficiency. Next, $60 \mu \mathrm{g}$ of Proteinase K (PK) were added and incubated for $2 \mathrm{~h}$ at $45^{\circ} \mathrm{C}$. Following PK digestion, $700 \mu \mathrm{M}$ of dibenzocyclooctyne-cy5 (DBCO-cy5) were added and incubated overnight at $37^{\circ} \mathrm{C}$. The Dual-labeled DNA was then embedded in low melting agarose gel plugs and washed in $10 \mathrm{ml}$ wash buffer (20 mM Tris-HCl, $50 \mathrm{mM}$ EDTA, $\mathrm{pH}$ 8) for $15 \mathrm{~min}$ 5 times and twice with TE $\mathrm{pH}$ 8. Plugs were melted at $70{ }^{\circ} \mathrm{C}$ for $2 \mathrm{~min}$ and incubated at $43{ }^{\circ} \mathrm{C}$ for 5 mins. $2 \mu$ of beta-agarase (Thermo Fisher Scientific) were added for agarose digestion and the reaction was incubated at $43{ }^{\circ} \mathrm{C}$ for $45 \mathrm{~min}$. Dual-labeled DNA was stained with DNA stain (BioNano Genomics) according to the Irys Prep NLRS protocol with the addition of $25 \mathrm{mM}$ Tris $\mathrm{pH} 8$ and $30 \mathrm{mM} \mathrm{NaCl}$. DNA concentration was measured by Qubit HS dsDNA assay.

To test non-methylation labelling efficiency, DNA was labelled with eM.SssI as described above, with or without MTAN. All DNA samples were purified from excess fluorophores, then applied on a custom epoxy-covered multi-well slide and imaged using commercial slide scanner, as described in the work of Margalit et $a l^{53}$. 


\section{Glass slide preparation and quality-control imaging}

$22 \times 22 \mathrm{~mm}^{2}$ glass cover-slips were cleaned for at least 7 hours to overnight by incubation in a freshly made $2: 1(\mathrm{v} / \mathrm{v})$ mixture of $70 \%$ nitric acid and $37 \%$ hydrochloric acid. After extensive washing with ultrapure water (18 M $\Omega$ ) and then with ethanol, cover slips were dried under a stream of nitrogen. Dry cover-slips were immersed in a premixed solution containing $750 \mu \mathrm{l} \quad \mathrm{N}$-trimethoxysilylpropyl-N,N,Ntrimethylammonium chloride and $200 \mu \mathrm{l}$ of vinyltrimethoxysilane in $300 \mathrm{ml}$ ultrapure water and incubated overnight at $65^{\circ} \mathrm{C}$. After incubation, cover-slips were thoroughly washed with ultrapure water and ethanol and stored at $4{ }^{\circ} \mathrm{C}$ in ethanol. The silane solution was freshly made and thoroughly mixed before the cover-slips were introduced into the mixture. Stored cover-slips were normally used within 2 weeks.

For quality-control, samples were imaged to evaluate DNA length and degree of labeling. Samples were diluted 1:100-1:300 in TE with DTT buffer (10 mM Tris pH 8, $1 \mathrm{mM}$ EDTA, $200 \mathrm{mM}$ DTT, Sigma) and were stained with $130 \mathrm{nM}$ YOYO-1 (Invitrogen) DNA intercalating dye. DNA molecules were stretched by placing $8 \mu 1$ of the solution at the interface of an activated coverslip placed on a standard microscope slide. The extended DNA molecules were imaged with a fluorescence microscope (TILL Photonics GmbH) using an Olympus UPlanApo 100X 1.3 NA oil immersion objective. Each image was composed of three colors, the YOYO-1, atto-532 and the Cy5 fluorophores, and was therefore imaged with the appropriate filters (485/20, 537/26 and 650/13 bandpass excitation filters, 525/30, 578/16 and 684/24 bandpass emission filters, for YOYO-1 and Cy5, respectively). Images were acquired by a DU888 EMCCD (Andor technologies) with an EM gain setting of 300 and exposure times of 100 for YOYO-1 and $2000 \mathrm{~ms}$ for atto-532 and Cy5.

\section{Global methylation quantification}

Differences in global fluorescence intensity following labeling with and without MTAN, and between genomic samples were analyzed by an in house software ${ }^{34,54}$ (https://github.com/ebensteinLab/Tiff16_Analyzer). The code measures the total length of each molecule as well as the number and intensity of $\mathrm{CpG}$ labels detected along the DNA molecules. Quantification of non-methylated $\mathrm{CpG}$ was done by dividing the total intensity of the CpG label by the total length of the DNA (calculated as intensity of methylation signal / total DNA length in kbp). Similar analysis was 
performed on images of DNA stretched in nanochannels from CLL patient and healthy donor. In total we sampled 5.585 Gbp from CLL patient and $5.865 \mathrm{Gbp}$ from the healthy donor and a total of 9840 field of view (FOV) from each donor.

\section{Optical mapping and analysis}

Loading of DNA in nanochannels and imaging were performed using an Irys instrument (BioNano Genomics). Detection of imaged molecules and fluorescent labels along each molecule was performed by AutoDetect (version 2.1.4, BioNano Genomics). Alignment to the reference genome was performed using Irys View software (version 2.3, BioNano Genomics). Arabidopsis accession col-0 was run on a single chip on the Irys platform (BioNano Genomics), for up to 120 cycles to collect up to $89 \mathrm{~Gb}$ of quality filtered data. Molecule files (.bnx) were loaded into IrysView, quality filtered (>100 kbp length, >2.75 signal/noise ratio) and aligned to the reference TAIR10 (https://www.arabidopsis.org/download/indexauto.jsp?dir=\%2Fdownload_files\%2FGenes\%2FTAIR10_genome_release).

Reference genome was converted into .cmap format and used to align the detected molecules using standardized parameters (-nosplit 2 -BestRef 1 -biaswt 0 -Mfast 0 -FP 1.5 -FN 0.15 -sf 0.2 -sd 0.0 -A 5 -outlier 1e-3 -outlierMax 40 -endoutlier 1e-4 -S -1000 -sr 0.03 -se 0.2 -MaxSF 0.25 -MaxSE 0.5 -resbias 464 -maxmem 64 -M 33 -minlen 100 -T 1e-6 -maxthreads 32 -hashgen 532.41 .50 .055 .0113 -hash -hashdelta 10 hashoffset 1 -hashmaxmem 64 -insertThreads 4 -maptype 0 -PVres 2 -PVendoutlier AlignRes 2.0 -rres 0.9 -resEstimate -ScanScaling 2 -RepeatMask 50.01 -RepeatRec 0.7 0.61 .4 -maxEnd 50 -stdout -stderr -usecolor 1). Alignments were analyzed for genome coverage, and gaps using the .xmap output file.

Following alignment, data was uploaded to IrysExtract ${ }^{38}$ to generate an average intensity profile of fluorescence intensity across the genome. We used all molecules aligned with a confidence equal or higher than $8\left(\mathrm{P}<=10^{-8}\right)$ and that had at least $70 \%$ of each molecule aligned to the reference. All regions in the genome with less than $15 \mathrm{x}$ coverage (3\% of the genome), were excluded from the data. The analysis pipeline results in a genome-wide intensity bedgraph file at $500 \mathrm{bp}$ resolution. The file allows further analysis and visualization in a genome browser as seen in Figures 4 \& 5 . 


\section{Comparison of optical methylation mapping to bisulfite sequencing}

Genome-wide bisulfite sequencing data $(\mathrm{GSE} 43857)^{36}$ was processed to generate a bedgraph track of non-methylated CpGs represented as a "methylation score". To calculate the methylation score, the non-methylated CpGs were padded $+/-500 \mathrm{bp}$ and summed over $1 \mathrm{kbp}$ bins to generate the bedgraph file. The score in each bin was then divided by the maximum score, setting the data between $0-1$. The genome was then divided to $10 \mathrm{kbp}$ windows and the mean score from each method (methylation score for the bisulfite data and relative intensity for the optical mapping data) was calculated for each window, K-S test was performed to test normality and since the data do not normally distribute, Spearman correlation analysis was performed using SPSS (IBM Corp. Released 2016. IBM SPSS Statistics for Windows, Version 24.0), to determine the linear dependence between bisulfite sequencing and methylation mapping.

\section{Analysis of CpG methylation distribution across gene body and siRNA}

pol-iv-dependent $24 \mathrm{nt}$ short interfering RNAs (siRNA) was retrieved from the previously published data ${ }^{41}$. miRNA primary transcript loci were extracted from miRBase ath.gff3 file (http://www.mirbase.org/ftp.shtml) ${ }^{55}$. Arabidopsis TAIR10 TAS loci were obtained from the ta-siRNA database ${ }^{56}$. The bed files of the 24 nt siRNA loci, miRNA precursor loci, and TAS loci were used to measure the non-methylated CpGs from optical mapping and genome-wide bisulfite sequencing as detailed below (supplementary file 5).

First, a file containing all CpGs was created from TAIR10 FASTA by knickers (version 1.5.5 Bionano Genomics). Then, a non-methylated CpG track from genome-wide bisulfite sequencing data of $A$. thaliana was created using BEDTools (version 2.25.0) subtract ${ }^{57}$. Each site in the non-methylated $\mathrm{CpG}$ track was expanded by 500 bp on both sides to adjust to the optical mapping resolution. Genome coverage of non-methylated $\mathrm{CpG}$ was generated by BEDTools genomecov to receive a non-methylated $\mathrm{CpG}$ bedgraph. Finally, this file was uploaded to deepTools ${ }^{58}$ in addition to the average label intensity from optical mapping and the clusters bed file. Using computeMatrix in reference point mode, we computed the mean value for each bin from all regions 10 kbp upstream and downstream from the clusters. All clusters were set to position 0 , and median coverage for the neighboring regions in $1 \mathrm{kbp}$ bins was plotted.

For gene body analysis, we compared the average label intensity of non-methylated CpGs from optical mapping and genome-wide bisulfite sequencing data with a bed file 
containing all A. thaliana genes (TAIR10) (supplementary file 6). Using computeMatrix in scale-region mode, all genes were scaled to the same length in 100 bp bins. We added $1500 \mathrm{bp}$ upstream and downstream to the TSS and TES (respectively) and computed the mean value for each bin.

Safety Statement. No unexpected or unusually high safety hazards were encountered.

\section{ASSOCIATED CONTENT}

Supporting information

Supplementary file 1- additional results as described in the main text (PDF)

Supplementary file 2- optical mapping intensity healthy blood sample.

Supplementary file 3- optical mapping intensity CLL sample.

Supplementary file 4- comparison between non- methylation $\mathrm{CpG}$ sites in optical mapping vs. bisulfite sequencing.

Supplementary file 5-24nt siRNA, miRNA precursor loci and TAS loci in A. thaliana. Supplementary file 6- genomic locations of A. thaliana genes.

\section{AUTHOR INFORMATION}

Y.E. and E.W. conceived the study. T.G performed optical mapping experiments and data analysis. Y.M, S.A and D.T performed experiments and data analysis. Z.S.Z provided MTAN enzyme. M.J and E.W provided M.Ssss I enzyme and cofactor. J.A.L and C.C. performed methylation analysis in Arabidopsis. N.A helped with human samples and clinical aspects. T.S, Y.E. and Y.M wrote the manuscript. All authors read and edited the manuscript.

Corresponding author:

* E-mail: uv@post.tau.ac.il, elmar.weinhold@oc.rwth-aachen.de

\section{ORCID}

Yuval Ebenstein: 0000-0002-7107-7529

Elmar Weinhold: 0000-0002-6481-2257 


\section{ACKNOWLEDGMENTS}

Y.E. acknowledges European Research Council consolidator grant (817811). Y.E. and E.W. acknowledge funding from a joint Israeli German R\&D Nanotechnology grant (Israel Innovation Authority, German Federal Ministry of Education and Research 13GW0282B and NATI 61976). Work in the Law laboratory was supported by an NIH NIGMS grant (GM112966) and a Salk Innovation Grant as well as the Rita Allen Foundation Scholars Program and the Hearst Foundation. C.C. was supported by a Postdoctoral Fellowship from the Glenn Center for Research on Aging at the Salk Institute and from a Salk Women and Science award. Z.S.Z was supported by NIH NIGMS (1R01GM101396)

We thank Kerstin Glensk for the preparation of eM.SssI. We thank Joseph R. Ecker and Florian Jupe for providing initial DNA samples.

\section{REFERENCES}

(1) Schumacher, A.; Kapranov, P.; Kaminsky, Z.; Flanagan, J.; Assadzadeh, A.;

Yau, P.; Virtanen, C.; Winegarden, N.; Cheng, J.; Gingeras, T.; et al.

Microarray-Based DNA Methylation Profiling: Technology and Applications.

2006, 34 (2), 528-542.

(2) Bird, A. DNA Methylation Patterns and Epigenetic Memory. Genes Dev. 2002, $16(1), 6-21$.

(3) Ehrlich, M.; Gama-sosa, M. A.; Huang, L.; Midgett, R. M.; Kenneth, C.;

Mccune, R. A.; Gehrke, C. Amount and Distribution of 5-Methylcytosine in Human DNA from Different Types of Tissues or Cells. Nucleic Acids Res. 1982, $10(8), 11-14$.

(4) Esteller, M. CpG Island Hypermethylation and Tumor Suppressor Genes: A Booming Present, a Brighter Future. Oncogene 2002, 21 (35), 5427-5440.

(5) Melanie Ehrlich. DNA Hypomethylation in Cancer Cells. Epigenomics 2009, 1 (2), 239-259.

(6) Ehrlich, M. DNA Methylation in Cancer: Too Much, but Also Too Little. 2002, 5400-5413.

(7) Brothman, A. R.; Swanson, G.; Maxwell, T. M.; Cui, J.; Murphy, K. J.;

Herrick, J.; Speights, V. O.; Isaac, J.; Rohr, L. R. Global Hypomethylation Is Common in Prostate Cancer Cells : A Quantitative Predictor for Clinical 
Outcome ? 2005, 156, 31-36.

(8) Seifert, H.; Schmiemann, V.; Mueller, M.; Kazimirek, M.; Onofre, F.;

Neuhausen, A.; Florl, A. R.; Ackermann, R.; Boecking, A.; Schulz, W. A.; et al. In Situ Detection of Global DNA Hypomethylation in Exfoliative Urine Cytology of Patients with Suspected Bladder Cancer. 2007, 82, 292-297.

(9) Zhang, H.; Lang, Z.; Zhu, J. Dynamics and Function of DNA Methylation in Plants. Nat. Rev. Mol. Cell Biol. 2018, 19 (August).

(10) Cokus, S. J.; Feng, S.; Zhang, X.; Chen, Z.; Merriman, B.; Haudenschild, C. D.; Pradhan, S.; Nelson, S. F.; Pellegrini, M.; Jacobsen, S. E. Shotgun

Bisulphite Sequencing of the Arabidopsis Genome Reveals DNA Methylation Patterning. Nature 2008, 452 (7184), 215-219.

(11) Lister, R.; O’Malley, R. C.; Tonti-Filippini, J.; Gregory, B. D.; Berry, C. C.; Millar, A. H.; Ecker, J. R. Highly Integrated Single-Base Resolution Maps of the Epigenome in Arabidopsis. Cell 2008, 133 (3), 523-536.

(12) Lister, R.; O’Malley, R. C.; Tonti-Filippini, J.; Gregory, B. D.; Berry, C. C.; Millar, A. H.; Ecker, J. R. Highly Integrated Single-Base Resolution Maps of the Epigenome in Arabidopsis. Cell 2008, 133 (3), 523-536.

(13) Li, P.; Demirci, F.; Mahalingam, G.; Demirci, C.; Nakano, M.; Meyers, B. C. An Integrated Workflow for DNA Methylation Analysis. J. Genet. Genomics 2013, 40 (5), 249-260.

(14) Lorthongpanich, C.; Cheow, L. F.; Balu, S.; Quake, S. R.; Knowles, B. B.; Burkholder, W. F. Single-Cell DNA-Methylation Analysis Preimplantation Embryos. Science (80-. ). 2013, 341 (September), 1110-1112.

(15) Mooijman, D.; Dey, S. S.; Boisset, J.-C.; Crosetto, N.; van Oudenaarden, A. Single-Cell 5hmC Sequencing Reveals Chromosome-Wide Cell-to-Cell Variability and Enables Lineage Reconstruction. Nat. Biotechnol. 2016, 34 (8), $852-856$.

(16) Treangen, T. J.; Salzberg, S. L. Repetitive DNA and Next-Generation Sequencing: Computational Challenges and Solutions. Nat. Rev. Genet. 2011, $13(1), 36-46$.

(17) Song, C.-X.; Clark, T. A.; Lu, X.-Y.; Kislyuk, A.; Dai, Q.; Turner, S. W.; He, C.; Korlach, J. Sensitive and Specific Single-Molecule Sequencing of 5Hydroxymethylcytosine. Nat. Methods 2011, 9 (1), 75-77.

(18) Flusberg, B. A.; Webster, D. R.; Lee, J. H.; Travers, K. J.; Olivares, E. C.; 
Clark, T. A.; Korlach, J.; Turner, S. W. Direct Detection of DNA Methylation during Single-Molecule, Real-Time Sequencing. Nat. Methods 2010, 7 (6), $461-465$.

(19) Rand, A. C.; Jain, M.; Eizenga, J. M.; Musselman-Brown, A.; Olsen, H. E.; Akeson, M.; Paten, B. Mapping DNA Methylation with High-Throughput Nanopore Sequencing. Nat. Methods 2017, 14 (4), 411-413.

(20) Simpson, J. T.; Workman, R. E.; Zuzarte, P. C.; David, M.; Dursi, L. J.; Timp, W. Detecting DNA Cytosine Methylation Using Nanopore Sequencing. Nat. Methods 2017, 14 (4), 407-410.

(21) Stoiber, M. H.; Quick, J.; Egan, R.; Lee, J. E.; Celniker, S. E.; Neely, R.; Loman, N.; Pennacchio, L.; Brown, J. B. De Novo Identification of DNA Modifications Enabled by Genome-Guided Nanopore Signal Processing. bioRxiv 2016, 094672.

(22) Dijk, E. L. Van; Jaszczyszyn, Y.; Naquin, D.; Thermes, C. The Third Revolution in Sequencing Technology. Trends Genet. 2018, 34 (9), 666-681.

(23) Gabrieli, T.; Sharim, H.; Nifker, G.; Jeffet, J.; Shahal, T.; Arielly, R.; LeviSakin, M.; Hoch, L.; Arbib, N.; Michaeli, Y.; et al. Epigenetic Optical Mapping of 5-Hydroxymethylcytosine in Nanochannel Arrays. ACS Nano 2018, 12 (7), $7148-7158$.

(24) Sharim, H.; Grunwald, A.; Gabrieli, T.; Michaeli, Y.; Margalit, S.; Dufault, B.; Chandra, S. Sen; Liu, A.; Bhattacharya, S.; Hastie, A. R.; et al. Long-Read Single-Molecule Maps of the Functional Methylome. Genome Res. 2019, 1-38.

(25) Levy-Sakin, M.; Grunwald, A.; Kim, S.; Gassman, N. R.; Gottfried, A.; Antelman, J.; Kim, Y.; Ho, S. O.; Samuel, R.; Michalet, X.; et al. Toward Single-Molecule Optical Mapping of the Epigenome. ACS Nano 2014, 8 (1), $14-26$.

(26) Cao, H.; Hastie, A. R.; Cao, D.; Lam, E. T.; Sun, Y.; Huang, H.; Liu, X.; Lin, L.; Andrews, W.; Chan, S.; et al. Rapid Detection of Structural Variation in a Human Genome Using Nanochannel-Based Genome Mapping Technology. Gigascience 2014, 3 (1), 34.

(27) Margalit, S.; Abramson, Y.; Sharim, H.; Manber, Z.; Bhattacharya, S.; Chen, Y.-W.; Vilain, E.; Barseghyan, H.; Elkon, R.; Sharan, R.; et al. Long Reads Capture Simultaneous Enhancer-Promoter Methylation Status for Cell-Type Deconvolution. bioRxiv 2021, 2021.01.28.428654. 
(28) Renbaum, P.; Razin, A. Mode of Action of the Spiroplasma CpG Methylase M.SssI. FEBS Lett. 1992, 313 (3), 243-247.

(29) Renbaum, P.; Abrahamove, D.; Fainsod, A.; Wilson, G. G.; Rottem, S. Cloning , Characterization, and Expression in Escherichia Coli of the Gene Coding for the CpG DNA Methylase From. 1990, 18 (4), 1145-1152.

(30) Kriukienè, E.; Labrie, V.; Khare, T.; Urbanavičiūtè, G.; Lapinaitė, A.; Koncevičius, K.; Li, D.; Wang, T.; Pai, S.; Ptak, C.; et al. DNA Unmethylome Profiling by Covalent Capture of CpG Sites. Nat. Commun. 2013, 4, 2190.

(31) Borchardt, R. T.; Wu, Y. S. Potential Inhibitors of S-AdenosylmethionineDependent Methyltransferases. 1. Modification of the Amino Acid Portion of S-Adenosylhomocysteine. J. Med. Chem. 1974, 17 (8), 862-868.

(32) Ronning, D. R.; Iacopelli, N. M.; Mishra, V. Enzyme-Ligand Interactions That Drive Active Site Rearrangements in the Helicobacter Pylori 5???Methylthioadenosine/S-Adenosylhomocysteine Nucleosidase. Protein Sci. 2010, 19 (12), 2498-2510.

(33) Baylin, S. B.; Jones, P. A. A Decade of Exploring the Cancer EpigenomeBiological and Translational Implications. Nature Reviews Cancer. Nature Publishing Group October 23, 2011, pp 726-734.

(34) Torchinsky, D.; Ebenstein, Y. Sizing Femtogram Amounts of DsDNA by Single-Molecule Counting. Nucleic Acids Res. 2016, 44 (2), e17.

(35) Wahlfors, J.; Hiltunen, H.; Heinonen, K.; Hamalainen, E.; Alhonen, L.; Janne, J. Genomic Hypomethylation in Human Chronic Lymphocytic Leukemia. Blood 1992, 80 (8), 2074 LP-2080.

(36) Kawakatsu, T.; Huang, S. C.; Jupe, F.; Weigel, D.; Nordborg, M.; Ecker, J. R.; Urich, M. A.; Castanon, R.; Nery, J. R.; Barragan, C.; et al. Epigenomic Diversity in a Global Collection of Arabidopsis Thaliana Accessions Resource Epigenomic Diversity in a Global Collection of Arabidopsis Thaliana Accessions. 2016, 492-505.

(37) Matzke, M. A.; Mosher, R. A. RNA-Directed DNA Methylation: An Epigenetic Pathway of Increasing Complexity. Nature Reviews Genetics. Nature Publishing Group May 8, 2014, pp 394-408.

(38) Arielly, R.; Ebenstein, Y. Irys Extract. Bioinformatics 2017, 0-0.

(39) Patel, D. J.; Jacobsen, S. E. Polymerase IV Occupancy at RNA-Directed DNA Methylation Sites Requires SHH1. Nature 2013, 498 (7454), 385-389. 
(40) Zhao, Y.; Chen, X. Non-Coding RNAs and DNA Methylation in Plants.

National Science Review. Oxford University Press 2014, pp 219-229.

(41) Zhou, M.; Palanca, A. M. S.; Law, J. A. Locus-Specific Control of the de Novo DNA Methylation Pathway in Arabidopsis by the CLASSY Family. Nat.

Genet. 2018, 50 (6), 865-873.

(42) Michaeli, Y.; Shahal, T.; Torchinsky, D.; Grunwald, A.; Hoch, R.; Ebenstein, Y. Optical Detection of Epigenetic Marks: Sensitive Quantification and Direct Imaging of Individual Hydroxymethylcytosine Bases. Chem. Commun.

(Camb). 2013, 49 (77), 8599-8601.

(43) Torchinsky, D.; Michaeli, Y.; Gassman, N. R.; Ebenstein, Y. Types by MultiColour Fluorescent Labelling $\uparrow$. 2019, No. PreCR mix, 11414-11417.

(44) Zirkin, S.; Fishman, S.; Sharim, H.; Michaeli, Y.; Don, J.; Ebenstein, Y. Lighting up Individual DNA Damage Sites by in Vitro Repair Synthesis. J. Am. Chem. Soc. 2014, 136 (21), 7771-7776.

(45) Gilat, N.; Tabachnik, T.; Shwartz, A.; Shahal, T.; Torchinsky, D.; Michaeli, Y.; Nifker, G.; Zirkin, S.; Ebenstein, Y. Single-Molecule Quantification of 5Hydroxymethylcytosine for Diagnosis of Blood and Colon Cancers. Clin. Epigenetics 2017, 9 (1).

(46) Issa, J.; Baylin, S. B.; Herman, J. G. REVIEW DNA Methylation Changes in Hematologic Malignancies : Biologic and Clinical Implications. 1997.

(47) Deschamps, S.; Zhang, Y.; Llaca, V.; Ye, L.; Sanyal, A.; King, M.; May, G.; Lin, H. A Chromosome-Scale Assembly of the Sorghum Genome Using Nanopore Sequencing and Optical Mapping Stéphane. Nat. Commun. 2018, 9 (4844).

(48) Michael, T. P.; Bryant, D.; Gutierrez, R.; Borisjuk, N.; Chu, P.; Zhang, H.; Xia, J.; Zhou, J.; Peng, H.; Baidouri, M. El; et al. Comprehensive Definition of Genome Features in Spirodela Polyrhiza by High-Depth Physical Mapping and Short-Read DNA Sequencing Strategies. 2017, 617-635.

(49) Jiao, W.; Accinelli, G. G.; Hartwig, B.; Kiefer, C.; Baker, D.; Severing, E.; Willing, E.; Piednoel, M.; Woetzel, S.; Madrid-herrero, E.; et al. Improving and Correcting the Contiguity of Long-Read Genome Assemblies of Three Plant Species Using Optical Mapping and Chromosome Conformation Capture Data. 2017, 778-786.

(50) Lukinavičius, G.; Tomkuvienè, M.; Masevičius, V.; Klimašauskas, S. 
Enhanced Chemical Stability of AdoMet Analogues for Improved

Methyltransferase-Directed Labeling of DNA. ACS Chem. Biol. 2013, 8 (6), 1134-1139.

(51) Cannon, L. M.; Butler, F. N.; Wan, W.; Sunny Zhou, Z. A Stereospecific Colorimetric Assay for (S,S)-Adenosylmethionine Quantification Based on Thiopurine Methyltransferase-Catalyzed Thiol Methylation. Anal. Biochem. 2002, 308 (2), 358-363.

(52) Lee, B. W. K.; Sun, H. G.; Zang, T.; Ju-Kim, B.; Alfaro, J. F.; Zhou, Z. S. Enzyme-Catalyzed Transfer of a Ketone Group from an S-Adenosylmethionine Analogue: A Tool for the Functional Analysis of Methyltransferases. J. Am. Chem. Soc. 2010, 132 (11), 3642-3643.

(53) Margalit, S.; Avraham, S.; Shahal, T.; Michaeli, Y.; Gilat, N.; Magod, P.; Caspi, M.; Loewenstein, S.; Lahat, G.; Friedmann-Morvinski, D.; et al. 5Hydroxymethylcytosine as a Clinical Biomarker: Fluorescence-Based Assay for High-Throughput Epigenetic Quantification in Human Tissues. Int. J. Cancer 2020, 146 (1), 115-122.

(54) Jain, N.; Shahal, T.; Gabrieli, T.; Gilat, N.; Torchinsky, D.; Vogel, V.; Ebenstein, Y. Global Modulation in DNA Epigenetics during ProInflammatory Macrophage Activation. Epigenetics 2019, 14 (12), 1183-1193.

(55) miRBase http://www.mirbase.org/ftp.shtml (accessed Nov 30, 2020).

(56) Zhang, C.; Li, G.; Zhu, S.; Zhang, S.; Fang, J. TasiRNAdb: A Database of TaSiRNA Regulatory Pathways. Bioinformatics 2014, 30 (7), 1045-1046.

(57) Quinlan, A. R.; Hall, I. M. BEDTools: A Flexible Suite of Utilities for Comparing Genomic Features. Bioinformatics 2010, 26 (6), 841-842.

(58) Ramírez, F.; Ryan, D. P.; Grüning, B.; Bhardwaj, V.; Kilpert, F.; Richter, A. S.; Heyne, S.; Dündar, F.; Manke, T. DeepTools2: A next Generation Web Server for Deep-Sequencing Data Analysis. Nucleic Acids Res. 2016, 44 (W1), W160-W165. 